\title{
Mineração
}

\section{Principais normas e recomendações existentes para o controle de vibrações provocadas pelo uso de explosivos em áreas urbanas - Parte II}

\author{
Denise de La Corte Bacci \\ Pós-doutoranda - LACASEMIN - Depart. de Engenharia de Minas e de Petróleo - Escola Politécnica - USP \\ E-mail:dbacci@dglnet.com.br \\ Paulo Milton Barbosa Landim \\ Depart. de Geologia Aplicada - Instituto de Geociências e Ciências Exatas - UNESP/Rio Claro (SP) \\ E-mail:plandim@rc.unesp.br \\ Sérgio Médici de Eston \\ Professor Titular e Chefe do Depart. de Engenharia de Minas e de Petróleo; Coordenador do Laboratório de \\ Controle Ambiental, Higiene e Segurança na Mineração (LACASEMIN) - Escola Politécnica - USP \\ E-mail:smeston@usp.br \\ Wilson Siguemasa Iramina \\ Pesquisador do LACASEMIN - Depart. de Engenharia de Minas e de Petróleo - Escola Politécnica - USP \\ E-mail:wilsiram@usp.br
}

\section{Resumo}

As atividades que envolvem o uso de explosivos devem ser controladas, não só com relação ao desmonte de estruturas (rocha e outros materiais), mas também quanto a danos estruturais em edificações próximas (casas, edificações históricas, etc.) e outros impactos ambientais como vibração, propagação de ruídos, ultralançamentos e sobrepressão atmosférica. Tais atividades são regidas por normas técnicas que sugerem parâmetros de medição e limites definidos na avaliação de prováveis danos. No caso específico de minerações em áreas urbanas, a velocidade de vibração de partícula $(\mathrm{Vp})$, normalmente expressa em $\mathrm{mm} / \mathrm{s}$, é o parâmetro que tem dado melhor correlação na avaliação de possíveis danos às estruturas civis, atribuídos às vibrações do terreno. As diferentes normas existentes apresentam valores de Vp que variam de $2 \mathrm{~mm} / \mathrm{s}$ para edifícios históricos até $150 \mathrm{~mm} / \mathrm{s}$ para construções em concreto armado. A maioria delas considera na avaliação de danos estruturais, além da velocidade, a freqüência da vibração. Algumas normas foram elaboradas com base em dados experimentais, analisando parâmetros como o tipo de construção e o material nela utilizados, outras se basearam apenas em valores empíricos, mas todas apresentam valores conservativos. A norma brasileira não avalia o parâmetro frequiência e não classifica os diferentes tipos de estruturas civis, restringindo-se ao valor resultante da velocidade de vibração como parâmetro medido, sendo, assim, limitada e deficiente em relação às normas internacionais. A coletânea aqui apresentada reuniu as normas nas Américas e em outros continentes, além de uma comparação com as normas européias mais importantes em âmbito mundial.

Palavras-chave: vibrações do terreno, explosivos, danos em estruturas, normas técnicas.

\section{Abstract}

Blasting requires control measures related to structural damage to buildings and environmental impacts like ground vibrations, noise, flyrock and air blast. The use of explosives is controlled by federal and state regulations, which involve measurement of parameters to evaluate probable damage in buildings and other type of constructions. In urban areas, the peak particle velocity (PPV) associated with ground vibration and expressed in $\mathrm{mm} / \mathrm{second}$, is the best parameter to evaluate possible structural damages. Worldwide legal limits vary from a low $2 \mathrm{~mm} / \mathrm{s}$ for historical buildings to a high $150 \mathrm{~mm} / \mathrm{s}$ for reinforced concrete.

Most of the regulations consider peak particle velocity and frequency as a double damage parameter. Some regulations were elaborated with an experimental database, involving different types of construction and building materials. Others were proposed using empirical data. Both regulations present conservative values. The Brazilian norm does not consider the frequency and the different types of buildings in the damage evaluation.

This paper presents a review of American and other regulations for blasting activities as compared to European regulations.

Keywords: ground vibrations, explosives, structural damage, regulations. 


\section{Introdução}

Os valores limites do nível de vibração do terreno não dependem apenas dos danos que a velocidade de vibração de partícula pode causar nas construções civis, mas também do tipo de construção em si, tendo sido provado que, com freqüência, a vibração gerada por explosivos é apenas o instante detonador de um processo de instabilidade atribuído a outras causas, como recalque, dilatação térmica, insuficiência de material, erro de cálculo de projeto, etc. (Fornaro, 1980).

Também é importante considerar as características próprias das vibrações, ou seja, a frequiência, a repetitividade e a duração do fenômeno. Pode-se dizer que um edifício sofre danos, se os impulsos dinâmicos provocados pelas vibrações sobrepõem-se aos impulsos estáticos, levando a uma superação das condições de resistência da estrutura.

Quando não é possível, partindo apenas das medidas de velocidade, atingir os valores de deslocamento e os impulsos, é necessário recorrer-se a tabelas empíricas de danos, correlacionando, de vários modos, as características mais evidentes do fenômeno. Esse é o caminho sugerido pela maior parte das normas (Fornaro, 1980).

Serão apresentadas, a seguir, as normas nas Américas, a norma australiana e a norma indiana relacionadas ao nível de vibração decorrente do uso de explosivos em minerações, além de uma comparação destas com as principais normas européias, indicando quais os parâmetros e os valores-limites de prováveis danos estruturais que cada uma delas apresenta.

\section{Norma Norte- Americana - USBM (RI 8507) e OSMRE}

O Bureau of Mines americano sempre se destacou como pioneiro nos estudos das vibrações, tendo como preocupação o estabelecimento de um limite de segurança que não causasse danos estruturais em construções civis.
A maioria dos seus trabalhos correlaciona os parâmetros deslocamento, freqüência, velocidade máxima de partícula e distância segura com a energia liberada na detonação.

Duvall e Fogelson (1962) concluíram que danos em residências são proporcionais à velocidade de vibração de partícula e que danos maiores (queda de reboco ou rachaduras) podem ser esperados a partir de Vp de $190 \mathrm{~mm} / \mathrm{s}$ (7,6pol/s). Já danos menores (trincas no reboco, abertura de rachaduras preexistentes) podem ser esperados a partir de $\mathrm{Vp}$ de $140 \mathrm{~mm} / \mathrm{s}(5,6 \mathrm{pol} / \mathrm{s})$ e que $50 \mathrm{~mm} / \mathrm{s}(2,0 \mathrm{pol} / \mathrm{s})$ representa um valor razoável de separação entre zona de segurança e uma zona de prováveis danos.

O Boletim 656, publicado pelo Bureau of Mines em 1971, intitulado "Blasting Vibrations and Their Effects of Structures", propôs uma velocidade máxima de partícula de $50 \mathrm{~mm} / \mathrm{s}(2,0 \mathrm{pol} / \mathrm{s})$ como o nível de segurança para as construções civis. A probabilidade de danos a uma estrutura residencial varia conforme aumenta ou diminui, em proporção, o nível de vibração acima ou abaixo de $50 \mathrm{~mm} / \mathrm{s}$.

O critério atual de danos desenvolvido pelo United States Bureau of Mines (USBM) baseia-se nas pesquisas realizadas em minerações a céu aberto e publicadas em 1980 no Report of Investigation RI 8507, intitulado "Structure Response \& Damage Produced by

Tabela 1 - Níveis seguros de velocidades de vibração da partícula para estruturas civis (Fonte: Bacci, 2000, adaptado de Siskind et al., 1980).

Tipo de estrutura $\mathrm{Vp}(\mathrm{mm} / \mathrm{s})$

\begin{tabular}{lcc}
\hline & $\begin{array}{c}\text { A baixas } \\
\text { freqüências } \\
\mathbf{f}<\mathbf{4 0 ~} \mathbf{~ H z}\end{array}$ & $\begin{array}{c}\text { A altas } \\
\text { freqüências } \\
\mathbf{f}>\mathbf{4 0 ~} \mathbf{~ H z}\end{array}$ \\
\hline $\begin{array}{l}\text { Casas modernas - paredes } \\
\text { interiores pré-moldadas em gesso, } \\
\text { sem revestimento }\end{array}$ & 19 & 50 \\
\hline $\begin{array}{l}\text { Casas velhas - paredes interiores } \\
\text { com gesso ou revestimento de } \\
\text { madeira }\end{array}$ & 12,7 & 50 \\
\hline
\end{tabular}

Ground Vibration from Surface Mine Blasting" (Siskind et al., 1980). Nesse trabalho, foi constatado que existe um sério problema com a ressonância estrutural, originada em resposta à vibração de baixa frequiência propagada no terreno, apresentando como resultado aumentos em deslocamentos e deformações, o que veio reforçar a idéia de que danos podem ser ocasionados pela freqüência.

Os limites de danos adotados no RI 8507 foram definidos para "danos cosméticos do tipo mais superficial", ou seja, fissuras internas que se desenvolvem em todas as residências, independentemente das vibrações geradas pela detonação de explosivos.

Os níveis de vibração de partícula seguros foram definidos como "níveis com improbabilidade de produzir fissuras no interior de residências ou quaisquer outros danos". Esses níveis são apresentados na Tabela 1 e são definidos como limites conservativos. Os valores foram muito criticados pela indústria das pedreiras por serem considerados desfavoráveis à produção.

O United States Bureau of Mines (USBM) e o Office for Surface Mining Reclamation (OSRME) estabeleceram dois critérios para o controle dos danos provocados pelas vibrações no terreno. Os dois critérios, mostrados na Figura 1, constituem uma referência de velocidade máxima de vibração de partícula $(\mathrm{Vp})$ em função da freqüência. 


\section{Norma Brasileira (NBR 9653)- Associação Brasileira de Normas Técnicas (ABNT)}

Desde 1983, a Associação Brasileira de Normas Técnicas (ABNT) vem coletando e analisando dados técnicos da bibliografia internacional e associandoos à experiência nacional, através da sua Comissão de Estudos CE - 18.205.02.

A experiência brasileira advém de trabalhos em pedreiras operando junto à periferia das grandes concentrações urbanas, em especial, no litoral paulista e na Grande São Paulo. Em média, a área urbanizada se constitui de residências modestas, construídas por uma população de baixa renda.

Esses trabalhos foram analisados estatisticamente, com os seguintes objetivos:

a) Caracterizar as condições médias de operação das pedreiras, que correspondem às condições econômicas favoráveis.

b) Caracterizar o nível de vibração correspondente àquelas condições econômicas de operação.

Observando-se as correlações existentes entre as variáveis envolvidas no fenômeno: carga máxima por espera (Q) e distância (D), velocidade de vibração de partícula (Vp) observadas ou medidas nos trabalhos realizados, conclui-se:

a) No caso geral (227 medições) para todos os tipos de rocha estudados (gnaisse, granito, calcário e basalto), não foram observados valores de velocidade de vibração de partícula (Vp) superiores a $15 \mathrm{~mm} / \mathrm{s}$, a partir de $200 \mathrm{~m}$ das detonações.

b) Para a faixa de valores de D (distância) inferiores a 200m, sugeriram-se limites do uso da carga máxima por espera (Q), de modo a não se excederem os valores de velocidade de vibração da partícula (Vp) em $15 \mathrm{~mm} / \mathrm{s}$, ou seja:

- para $140<\mathrm{D}<200=>\mathrm{Q}<100 \mathrm{~kg} /$ espera

- para $40<\mathrm{D}<140=>\mathrm{Q}<30 \mathrm{~kg} /$ espera
Com base nas análises realizadas, a CE - 18.205.02 redigiu e aprovou a norma NBR 9653, que estabelece a velocidade de vibração de partícula (Vp) igual a $15 \mathrm{~mm} / \mathrm{s}$ como limite máximo de vibração admissível nos arredores da área de operação das pedreiras. A norma também estabelece que não devem ocorrer, de forma alguma, ultralançamentos de fragmentos e sobrepressões atmosféricas excessivas.

A velocidade resultante de vibração de partícula deve ser calculada com base na seguinte fórmula:

$$
\mathrm{VR}=\left[(\mathrm{VL})^{2}+(\mathrm{VT})^{2}+(\mathrm{VV})^{2}\right]^{1 / 2}
$$

onde:

$\mathrm{VR}=$ velocidade resultante de vibração da partícula, em mm/s.

$\mathrm{VL}=$ velocidade de vibração na direção longitudinal, em mm/s.

VT = velocidade de vibração na direção transversal, em mm/s.

VV = velocidade de vibração na direção vertical, em $\mathrm{mm} / \mathrm{s}$.

Também é definido o nível de sobrepressão atmosférica, medido além da área de operação, não devendo ultrapassar o valor de $134 \mathrm{dBL}$ pico.

Embora seja amplamente difundido que, para frequiências altas, a estrutura suporta melhor as vibrações, como no caso de estruturas fundadas em rocha localizadas a menos de 300m da detonação (Siskind et al., 1980), a norma brasileira não trata da freqüência dos fenômenos vibratórios, nem determina os tipos de edifícios afetados pelas vibrações, sendo, desse ponto de vista, deficiente em relação às normas internacionais já mencionadas.

Trabalhos de Langfors e Kihlstrom (1963) e do USBM (Siskind et al.,1980) indicam total ausência de danos nas condições anteriormente mencionadas e também para valores de $\mathrm{Vp}$ menores que $50 \mathrm{~mm} / \mathrm{s}$. Para uma faixa de freqüência entre 2 e $40 \mathrm{~Hz}$, os limites de Vp possuem ampla dispersão, devido aos grandes deslocamentos que ocorrem associados à ressonância estrutural (as freqüências naturais das estruturas estão nessa faixa). Para esses casos, os limites de velocidade de vibração de partícula (Vp) com ausência de danos em edifícios foram calculados por simples conversão de movimentos harmônicos simples, para a faixa de 13 a $25 \mathrm{~mm} / \mathrm{s}$, a $10 \mathrm{~Hz}$.

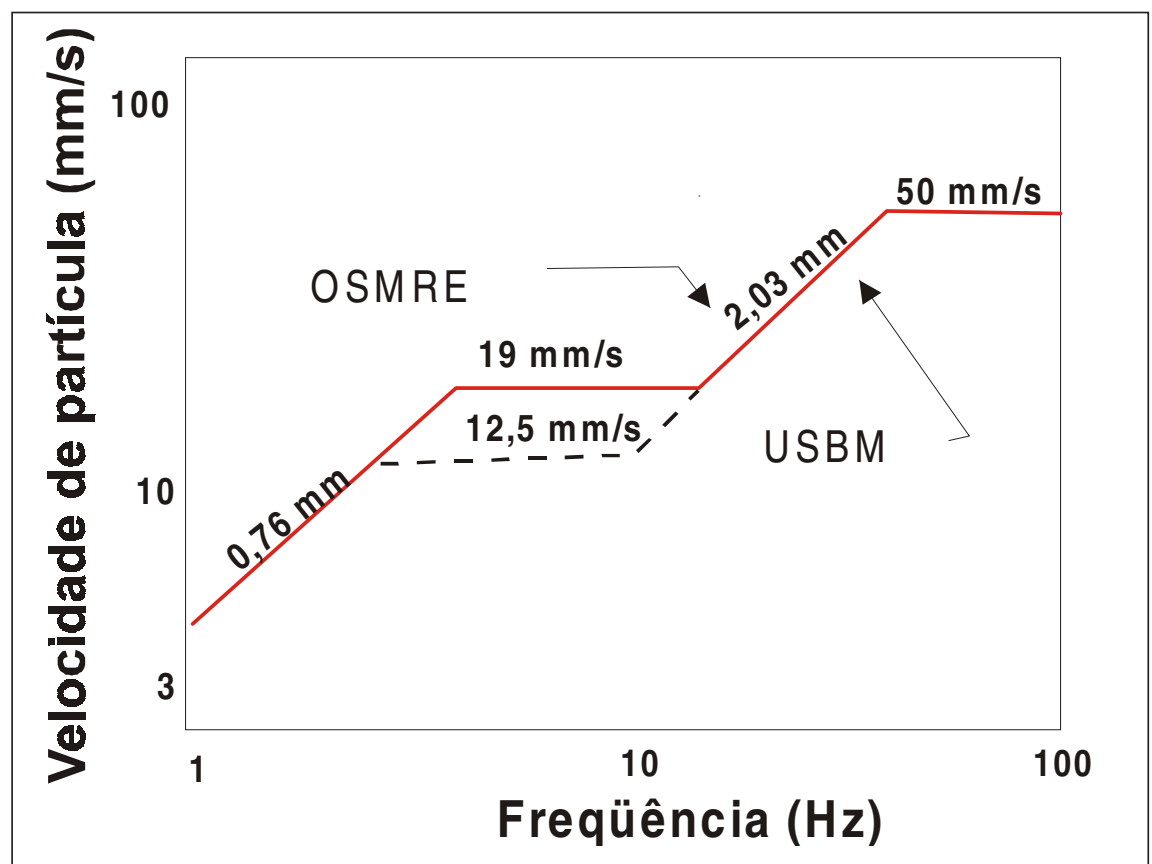

Figura 1 - Diagrama representando os limites de $V p$ e de deslocamento, sugeridos pelo USBM e OSMRE, medidos em $\mathrm{mm} / \mathrm{s}$ e $\mathrm{mm}$, respectivamente, em função da freqüência, em Hz. A linha tracejada, em baixo,refere-se aos valores propostos pelo USBM para paredes rebocadas. (Fonte: Bacci, 2000, modificado de Berta, 1985). 


\section{CETESB D7.013}

A CETESB estabeleceu critérios de avaliação de problemas ambientais através da norma interna D7.013, de 1992, na qual se fixam as condições exigíveis para a atividade de mineração a céu aberto que utiliza explosivos no desmonte do minério, no que se refere ao controle de poluição e à conservação do meio ambiente. Esse critério avalia o incômodo gerado à população e não se refere aos danos em estruturas civis, como a NBR 9653.

A velocidade resultante de vibração de partícula é calculada do mesmo modo que na norma NBR 9653, a qual estabelece como valor máximo de $\mathrm{Vp}$ $3 \mathrm{~mm} / \mathrm{s}$, medido na componente vertical. Quando a medição for realizada com utilização de instrumentos cujos resultados sejam a integração das três componentes, o valor máximo permitido para a velocidade de partícula é de 4,2 $\mathrm{mm} / \mathrm{s}$, para que não haja incômodo à população. Em ambos os casos, a medição deve ser feita fora dos limites da propriedade da mineração.

Quanto à instalação dos sensores, estes devem ser fixados rigidamente no solo, a uma distância inferior a $10 \mathrm{~m}$ da edificação mais atingida.

\section{Norma Australiana}

Os critérios australianos para limitar os níveis de vibração a partir de desmontes com explosivos em rochas são baseados nas especificações dos seguintes órgãos:

- Comissão Estadual de Controle da Poluição (SPCC) do Manual de Controle de Perturbação Ambiental (New South Wales) - 1980.

- Conselho Ambiental Australiano (AEC) - Norma AS2187, Parte 2 de 1983 - uso de explosivos.

- U.S. Bureau of Mines (USBM) - Relatório RI 8485.

O critério adotado pela SPCC para velocidade de vibração da partícula era o de não exceder $7 \mathrm{~mm} / \mathrm{s}$ e as detonações deveriam ser realizadas no período das 9 às 15 horas, para se evitarem inversões térmicas, sendo requisitado o monitoramento de todos os desmontes de um empreendimento.

A norma AS2187 (1983) adotou critérios referentes ao pico de velocidade de vibração da partícula, medido no terreno próximo à fundação da estrutura, como mostra a Tabela 2.

A norma foi reeditada em 1993, não considerando limites de Vp para as construções históricas e monumentos, mas ressaltando que esses requerem considerações especiais, muitas vezes resultado em medições adicionais na sua própria estrutura.

Em abril de 1986, o Environmental Noise Control Committee of the Australian Environmental Council (AEC) editou um documento intitulado "Draft Technical Basis for the Control of Noise and Vibration from Blasting", sugerindo as seguintes restrições:

a) Nível máximo permitido de velocidade de vibração da partícula de $5 \mathrm{~mm} / \mathrm{s}$.

b) O nível máximo pode ser excedido em $5 \%$ dos desmontes em um período de 12 meses, não ultrapassando nunca o valor de $10 \mathrm{~mm} / \mathrm{s}$.

c) Recomenda o valor de $2 \mathrm{~mm} / \mathrm{s}$ para o controle das vibrações.

d) Os desmontes são permitidos das 9 às $15 \mathrm{~h}$, de segunda à sábado, sendo proibidos nos domingos e feriados.

Tabela 2 - Valores máximos de velocidade de vibração da partícula, adotados pela Norma AS2187, segundo os tipos de construções civis (Fonte: Bacci, 2000, adaptado de Scott, 1996).

\begin{tabular}{l|c}
\hline \multicolumn{1}{c|}{ Tipos de construções } & $\begin{array}{c}\text { Velocidade máxima de } \\
\text { vibração de partícula } \\
\text { (mm/s) }\end{array}$ \\
\hline $\begin{array}{l}\text { Construções históricas e monumentos de } \\
\text { especial valor ou significado }\end{array}$ & 2 \\
\hline Casas e prédios residenciais de baixa altura & 10 \\
\hline $\begin{array}{l}\text { Prédios comerciais ou industriais ou } \\
\text { estruturas de concreto armado ou ferro }\end{array}$ & 25 \\
\hline
\end{tabular}
de uma vez ao dia, excetuando-se desmontes secundários.

f) As restrições quanto ao tempo e à frequiência dos desmontes não se aplicam a locais onde os efeitos das vibrações não são perceptíveis e a minas subterrâneas metalíferas.

\section{Norma Indiana}

O limite de vibração suportado pelas estruturas civis na Índia nos arredores das minas a céu aberto foi determinado pelo Central Mining Research Institute (CMRI), em 1991, através de um monitoramento da resposta estrutural de diversas construções.

A Tabela 3 apresenta os resultados de tal estudo.

Os estudos apontam que um valor de $\mathrm{Vp}$ de $5 \mathrm{~mm} / \mathrm{s}$, medido na fundação das estruturas, é seguro a baixas freqüências para qualquer tipo de estrutura residencial, mesmo se este se amplifica no nível mais alto, devido à ressonância.

A Tabela 4 mostra os valores mínimos de vibração e os danos que podem ser gerados nas estruturas, segundo estudos do CMRI, em diversos tipos de residências.

Os valores propostos nesse estudo foram monitorados em residências, escolas, construções pobres e demais e) Os desmontes não podem ocorrer mais 
locais de interesse. Muitas dessas residências encontravam-se em mau estado de conservação e foram construídas sem nenhuma supervisão e com material de baixa qualidade.

\section{Análise das principais normas técnicas}

As principais diferenças entre as normas americanas em relação às recomendações européias são:

a) Quanto à instalação do geofone: na norma americana os geofones são, em geral, dispostos no terreno circundante à estrutura em observação, e não no interior do edifício ou em correspondência de suas partes particularmente sensíveis.

b) Quanto aos tipos de edifícios: as recomendações do USBM distinguem os edifícios em duas classes, considerando o tipo de revestimento interno, ou seja, aqueles construídos com paredes rebocadas, e os construídos sem reboco nas paredes, ou com revestimentos em gesso ou madeira. Os limites para as paredes à vista (sem reboco), propostos pelo USBM, coincidem com aqueles do OSMRE, no campo da freqüência compreendido entre $0 \mathrm{e}$ $11 \mathrm{~Hz}$ e entre 40 e $100 \mathrm{~Hz}$. A recomendação do OSRME permite maiores valores de Vp no intervalo de freqüência compreendido entre 11 e $40 \mathrm{~Hz}$. As normas européias definem o tipo de edifício em função do seu uso (residencial, industrial, histórico), dando valores de Vp mais baixos que a norma americana para frequiências menores que $40 \mathrm{~Hz}$. No intervalo de 40 a $100 \mathrm{~Hz}$, para edifícios industriais, os valores se aproximam daqueles da norma americana, mantendo-se mais baixos para os outros tipos de edifícios.

c) Quanto aos intervalos de frequiência: as normas norte-americanas definem os intervalos em menor e maior que $40 \mathrm{~Hz}$, pois a ressonância natural dos edifícios está abaixo desse valor. As normas européias definem intervalos de freqüência menores, devido aos tipos de construções mais antigas e, portanto, mais sensíveis encontradas nesses países.

Segundo Schillinger (1994), uma comparação entre a norma alemã DIN 4150 e a norma norte-americana USBM RI8507 mostra uma variação dos valores de $\mathrm{Vp}$ admitidos, como mostra a Figura 2. A razão entre a USBM RI8507 e a DIN 4150, para residências e monumentos históricos, mostra um fator 3 a 4 vezes maior para a norma americana, no intervalo de freqüência de 1 a $100 \mathrm{~Hz}$, significando que as estruturas residenciais dos Estados Unidos seriam de 3 a 4 vezes

Tabela 3 - Valores-limites estabelecidos pelo CMRI para vibrações na fundação a diferentes níveis de freqüência (Fonte: adaptado de Pal Roy, 1998).

\begin{tabular}{|c|c|c|c|}
\hline \multirow[t]{2}{*}{ Tipo } & \multirow[t]{2}{*}{ Especificações da estrutura } & \multicolumn{2}{|c|}{$\begin{array}{l}\text { Valores de Vp } \\
\quad(\mathrm{mm} / \mathrm{s})\end{array}$} \\
\hline & & $<24 \mathrm{~Hz}$ & $>24 \mathrm{~Hz}$ \\
\hline (a) & $\begin{array}{l}\text { Construções domésticas, paredes interiores } \\
\text { pré-moldadas em gesso; estruturas com } \\
\text { reboco; pontes. }\end{array}$ & 5 & 10 \\
\hline (b) & $\begin{array}{l}\text { Prédios industriais, estruturas de concreto } \\
\text { armado ou aço. }\end{array}$ & 12,5 & 25 \\
\hline (c) & $\begin{array}{l}\text { Estruturas de importância histórica; estruturas } \\
\text { muito sensíveis; com mais de } 50 \text { anos de } \\
\text { idade; estruturas em baixo estado de } \\
\text { conservação e sem reparações. }\end{array}$ & 2 & 5 \\
\hline
\end{tabular}

Tabela 4 - Valores mínimos de vibrações produzidas por desmontes nos quais ocorreram danos, medidos nas estruturas, segundo CMRI (1991).

\begin{tabular}{cccc}
\hline & Vp (mm/s) & Local de medição & Tipo de estrutura \\
\hline Ocorrência das primeiras trincas & 50 & Solo & Estrutura de tijolo \\
Ocorrência das primeiras trincas & 76 & Primeiro andar & Estrutura de tijolo \\
Ocorrência das primeiras trincas & 400 & Paredes laterais & Estrutura de concreto \\
Alargamento de trincas existentes & 90 & Solo & Estrutura de tijolo \\
Rachaduras & 240 & - & Estrutura de tijolo \\
Queda de reboco & 280 & - & Estrutura de tijolo \\
Fissuras profundas & 192 & Paredes laterais e cantos & Construções de sapê \\
\end{tabular}

REM: R. Esc. Minas, Ouro Preto, 56(2): 131-137, abr. jun. 2003135 
mais resistentes à cargas dinâmicas, que as construções européias. No entanto, o próprio autor não aceita essa hipótese e argumenta que as regulamentações possuem um caráter conservativo e não estão isentas de referências políticas. Tais referências, geralmente, estão concentradas na determinação da probabilidade de aceitação social da ocorrência de danos e incômodo. Atualmente, o incômodo aos seres humanos vem sendo até mais considerado que os danos em edifícios, embora com maior dificuldade para serem regulamentados, devido à sua subjetividade.

A norma brasileira não apresenta uma definição dos valores de Vp em relação à frequiência e não define os tipos de construção civil nem os possíveis danos que podem ocorrer em função do tipo de material utilizado. O valor definido pela ABNT como limite máximo de vibração a ser gerado é mais coerente com os estudos internacionais e não com a realidade das construções nacionais.

A CETESB apresenta valores mais rígidos para o limite de vibração de partícula, pois considera o incômodo ao ser humano e não os danos possíveis de serem causados em determinados tipos de estruturas civis, como define a NBR 9653.

A realidade brasileira mostra que a maioria das pedreiras em áreas urbanas é circundada por construções residenciais de baixa renda, na periferia dos grandes centros urbanos. Essas residências apresentam diversos tipos de problemas já na sua construção, como quedas de rebocos, trincas e rachaduras de paredes, originados, não pelas detonações com explosivos realizadas pelas pedreiras, mas por outros problemas como os de recalque do terreno, infiltração de água e, ainda, pela má qualidade do material utilizado. O maior problema das reclamações contra as pedreiras ocorre devido à sobrepressão atmosférica e ao ruído gerado na detonação e não pela vibração do terreno.

Quando se consideram os terrenos graníticos ou basálticos - que perfazem a maioria das jazidas próximas dos centros urbanos -, observa-se que os valo- res de vibração dificilmente excedem a norma NBR 9653, embora em alguns casos excedam os valores estabelecidos pela CETESB. Nesse caso, apresentam altas freqüências diminuindo muito o risco de possíveis danos estruturais, segundo os padrões internacionais. $\mathrm{O}$ in- cômodo gerado nesses casos é o que mais preocupa, restringindo os valores de Vp.

As Tabelas 5 e 6 comparam os parâmetros e os valores de velocidade e freqüiência das principais normas.

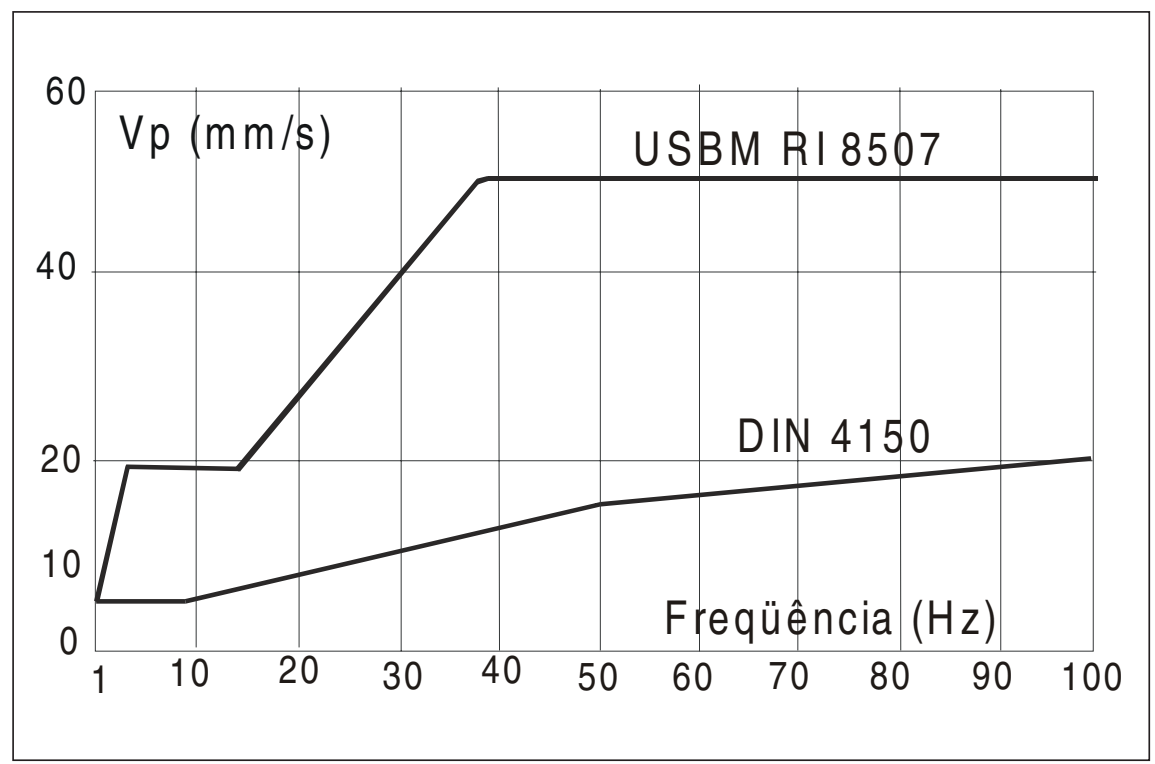

Figura 2 - Gráfico comparativo das normas alemã DIN 4150 e norte-americana RI 8507 (Fonte: Bacci, 2000, modificado de Schillinger, 1994).

Tabela 5 - Valores de velocidade de vibração de partícula e freqüência das principais normas internacionais. Mínimos e máximos estabelecidos em função do tipo de edifício.

\begin{tabular}{c|c|c|c|c}
\hline \multirow{2}{*}{ Países } & \multicolumn{2}{|c|}{ Velocidade $(\mathbf{m m} / \mathbf{s})$} & \multicolumn{2}{c}{ Freqüência $(\mathbf{H z})$} \\
\cline { 2 - 5 } & Mínima & Máxima & Mínima & Máxima \\
\hline Alemanha & 3 & 50 & $<10$ & 100 \\
\hline Estados Unidos & 12,7 & 50 & $<40$ & $>40$ \\
\hline Itália & 3 & 50 & $<10$ & 100 \\
\hline Suíça & 10 & 90 & 8 & 30 \\
\hline França & $2,5 / 4,0$ & $75 / 15$ & $<10 / 4$ & $>10 / 100$ \\
\hline Portugal & 2,5 & 60 & $<10$ & $>40$ \\
\hline Suécia & 18 & 70 & $<40$ & $>40$ \\
\hline Inglaterra & 15 & 50 & 4 & $>40$ \\
\hline Austrália & 2 & 25 & $<40$ & $>40$ \\
\hline Índia & 2 & 25 & $<24$ & $>24$ \\
\hline Brasil & - & 15 & - & $>40$ \\
\hline
\end{tabular}


Tabela 6 - Normas e recomendações para os edifícios.

\begin{tabular}{|c|c|c|c|c|c|c|c|c|c|}
\hline \multirow[b]{2}{*}{ Países } & \multirow[b]{2}{*}{ Normas } & \multicolumn{8}{|c|}{ Parâmetros } \\
\hline & & $\mathbf{F}$ & $\begin{array}{l}\text { Vel. } \\
\text { Total }\end{array}$ & $\begin{array}{c}\mathrm{Vp} \\
\text { Componente }\end{array}$ & Distância & Terreno & $\begin{array}{l}\text { Tipo de } \\
\text { Edifício }\end{array}$ & $\begin{array}{c}\text { Tipo de } \\
\text { Vibração }\end{array}$ & $\begin{array}{c}\text { Tipo de } \\
\text { Atividade }\end{array}$ \\
\hline Alemanha & DIN 4150 & Sim & Não & Sim & Não & Não & Sim & $\mathrm{I}, \mathrm{PI}$ & Não \\
\hline \multirow{2}{*}{ Estados Unidos } & USBM (1980) & Sim & Não & Sim & Não & Não & Sim & $\mathrm{I}, \mathrm{PI}$ & Não \\
\hline & OSMRE & Sim & Não & Sim & Não & Não & Sim & $\mathrm{I}, \mathrm{PI}$ & Sim \\
\hline Itália & UNI 9916 & Sim & Não & Sim & Não & Não & Sim & $\mathrm{I}, \mathrm{PI}$ & Não \\
\hline Suíça & SN640312/92 & Sim & Sim & Não & Não & Não & Sim & I, C & Não \\
\hline \multirow{3}{*}{ França } & AFTES 1974 & Sim & Não & Sim & Não & Sim & Sim & $\mathrm{I}, \mathrm{PI}$ & Não \\
\hline & Min.Ambiente & Sim & Não & Sim & Não & Não & Sim & I, PI, C & Sim \\
\hline & (GFEE) & Sim & Não & Sim & Não & Não & Sim & I & Sim \\
\hline Portugal & NP 2074 & Sim & Sim & Sim & Não & Sim & Sim & 1 & Sim \\
\hline Espanha & UNE 22-381 & Sim & Não & Sim & Não & Não & Sim & $\mathrm{I}, \mathrm{PI}$ & Não \\
\hline Suécia & SS4604866 & Sim & Não & Sim & Sim & Sim & Sim & $\mathrm{I}, \mathrm{PI}$ & Sim \\
\hline Finlândia & Min. Sanità & Sim & Não & Sim & Sim & Sim & Sim & I, PI, C & Não \\
\hline Austrália & AS2187 & Sim & Não & Sim & Não & Não & Sim & $\mathrm{I}, \mathrm{PI}$ & Sim \\
\hline Índia & CMRI & Sim & Sim & Sim & Não & Não & Sim & 1 & Não \\
\hline Brasil & NBR 9653 & Não & Não & Sim & Sim & Não & Não & $\mathrm{I}, \mathrm{PI}$ & Não \\
\hline
\end{tabular}

I - Impulsiva; PI - Periódica Impulsiva: C - Contínua.

\section{Referências}

\section{Bibliográficas}

ASSOCIAÇÃO BRASILEIRA DE NORMAS TÉCNICAS (ABNT). NBR 9653. Guia para avaliação dos efeitos provocados pelo uso de explosivos nas minerações em áreas urbanas. Norma de Procedimento.1986. São Paulo (SP).

BACCI, D.C. Vibrações geradas pelo uso de explosivos no desmonte de rochas: avaliação dos parâmetros físicos do terreno e dos efeitos ambientais. Rio Claro: Instituto de Geociências e Ciências Exatas, Universidade Estadual Paulista. 2000. v.1 (texto) e v.2 (anexos). (Tese de Doutorado).

BERTA, G. L'esplosivo strumento di lavoro. 1985. ITALESPLOSIVI - Milano. $1^{\text {a }}$ ed. 488p. Surface mineral extractions (except coal) sites. British Standard.
CETESB - COMPANHIA DE TECNOLOGIA DE SANEAMENTO AMBIENTAL Norma D7.013 - Mineração por explosivo. São Paulo: 1992. 7 p.

DUVALL, W.I., FOGELSON, D.E. Review of criteria for estimating damage to residences from blasting vibration. BuMines RI 5968, 1962. 19 p.

LANGEFORS, U., KIHLSTROM. The modern technique of rock blasting. New York, and Almqvist \& Wiksell, Stockholm: John Wiley \& Sons, Inc. 1963.

PAL ROY, P. Characteristics of ground vibrations and structural response to surface and underground blasting. Geotechnical and Geological Engineering, v. 16, p.151166, 1988.

REIHER, H., MEISER, F.J. Human annoyance of vibrations. Forschung auf dem Gebeite des Ingenieurwesens, v. 2, n. 11, 1931.
SCHILLINGER, R.R. Blasting vibrations and other environmental effects of blasting works. EXPLOSIVES 94 - CONFERENCE PROCEEDINGS - University of Leeds, U.K. - The Institute of Explosives Engineers. The European Federation of Explosives Engineers \& The University of Leeds Departament of Mining \& Mineral Engineering. 1994. p. 15-26.

SCOTT, A. Open pit blast design. In: JKMRC T.J. Napier Munn Editor. Austrália: The University of Queensland. 1996. 339 p.

SISKIND, D.E., STAGG, M.S., KOPP, J.W., DOWDING, C.H. Structure response and demage produced by ground vibration from surface mine blasting. Report of Investigation 8507 USBM - United States Bureau of Mines, 1980. 74 p.

Artigo recebido em 06/11/2002 e aprovado em 15/02/2003.

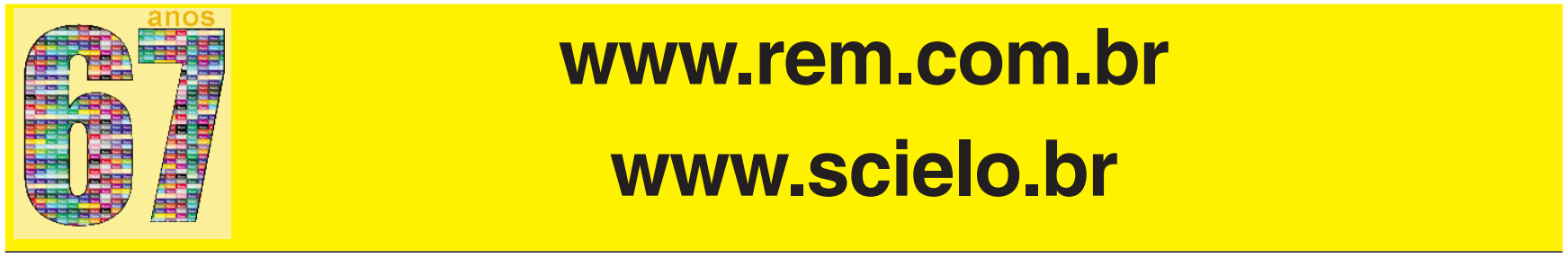

